\title{
Original
}

\section{Glial Fibrillary Acidic Protein in the Perivascular Glia of the Developing Human Retina}

\author{
Mohammed Mohibul KARIM ${ }^{1)}$ and Hiroshi ITOH $^{2)}$
}

\begin{abstract}
The expression of glial fibrillary acidic protein (GFAP) in the perivascular glia of the developing human retina was studied immunohistochemically in 22 normal eyes. Retinas were stained with anti-GFAP antibody using the labelled streptavidin-biotin method. The GFAP-positive perivascular glial cells were first observed in the human retina at 24 gestational weeks near the optic disc. As the retina matured, the intensity of GFAP immunoreactivity increased until full term. The GFAP immunoreactivity was most intense around large vessels near the optic disc and diminished with decreasing vascular caliber and increasing distance from the optic disc. Our results support the concepts that in the developing human retina, GFAP-positive perivascular glial cells first appear at 24 gestational weeks and that the human retina contains perivascular glial cells.
\end{abstract}

Key words: glial fibrillary acidic protein, immunohistochemistry, perivascular glia, retina, human

\section{Introduction}

In the human retina, astroctyes completely sheathe small blood vessels with their suckerlike end-feet. The special cellular element between this sheath and the vascular structures has been described as perivascular glia $^{1,2}$. A close relationship is believed to exist between the blood vessels of the retina ${ }^{1,2)}$ and brain $^{3)}$, and the glial cells. Despite numerous investigations, hte significance of this association is still unclear. Some investigations, the significance of this association is still unclear. Some investigators have shown that the astroctyes and their perivascular end-feet take part in the formation of the blood-brain barrier in the central nervous system $(\mathrm{CNS})^{4,5)}$.

In previous immunohistochemical and biochemical studies the various aspects of astrocytes have been described including their morphologic classes, development, functions, and distribution in the CNS and retina ${ }^{6-18}$. Glial fibrillary acidic protein (GFAP) is reported to be a major protein component of the glial filaments that are found in high concentrations in astrocytes ${ }^{19,20)}$. However, expression of GFAP in perivascular glial cells has not been well studied. The GFAP in the perivascular glia of the rat retina has been described by some authors $^{21)}$ and in the cortical perivascular glia of the cat by others ${ }^{22)}$. In this study, an antiGFAP antibody was used as a glial marker and the development and distribution of GFAPpositive perivascular glial cells was investigated in the human retina by utilizing the labelled

1) Department of Ophthalmology, Kobe University School of Medicine, Kusunoki-cho 7-5-1, Chuo-ku, Kobe 650, Japan.

2) First Department of Pathology, Kobe University School of Medicine. 
Table 1. Perivascular GFAP immunohistochemical reaction patterns of the retina.

\begin{tabular}{clll}
\hline \multicolumn{1}{c}{ Age } & \multicolumn{1}{c}{ NFL* } & \multicolumn{1}{c}{ GCL* } & IPL* \\
\hline $13-22 \mathrm{GW} \dagger$ & No & No & No \\
$24 \mathrm{GW} \dagger$ & Weak & Weak & No \\
$25-28 \mathrm{GW} \dagger$ & Larger area & Larger area & No \\
$30-37 \mathrm{GW} \dagger$ & Strong & Strong & No \\
$40 \mathrm{GW} \dagger$ & Larger area & Larger area & No \\
$4 \mathrm{Mo} \dagger$-Adult & Larger area & Larger area & Weak \\
\hline
\end{tabular}

* NFL, Nerve fiber layer; GCL, ganglion cell layer; IPL, inner plexiform layer

$\dagger \mathrm{GW}-$ Gestational weeks, Mo-month.

streptovidin-biotin (LSAB) method.

\section{Materials and Methods}

Twenty-two formalin-fixed normal human eyes ranging in age from 13 gestational weeks (GW) to adulthood were collected from the files of the First Department of Pathology, Kobe University School of Medicine, for immunohistochemical analysisc (Table 1). The tenets of the Declaration of Helsinki were folowed. All persons and authorities gave their informed consent prior to inclusion in this study. Eyes were cut anteroposteriorly parallel to the optic axis, and a strip of retina was taken from the temporal half of the globe excluding the macula and fovea. The strip was then transferred to cold phosphate-buffered saline (PBS) until processing for paraffin embedding was begun. Tissues were sectioned at $5 \mu \mathrm{m}$ parallel to the axis of the optic nerve. Sections were mounted on clean slides utilizing a gelatin water bath and dried overnight at 45 to $47^{\circ} \mathrm{C}$.

Rabbit anti-GFAP (primary antibody), purified from bovine spinal cord, was supplied by Dako Corporation (Carpinteria, CA, USA). This antibody reacts strongly with human GFAP and cross-reacts with GFAP in several other mammalian species. Biotinylated antirabbit and anti-mouse immunoglobulins were also supplied by Dako Corporation. Tissue sections were stained for anti-GFAP using the LSAB method (Dako LSAB kit, peroxidase). Endogenous peroxidase activity was abolished by incubation in $3 \%$ hydrogen peroxide for 5 minutes. Nonspecific staining was blocked by a 5-minute incubation with blocking reagent ( $15 \mathrm{ml}$ PBS containing carrier protein and $15 \mathrm{mM}$ sodium azide). Then, the specimen was incubated with appropriately diluted rabbit primary antibody $(7 \mathrm{ml}$ rabbit antiserum to

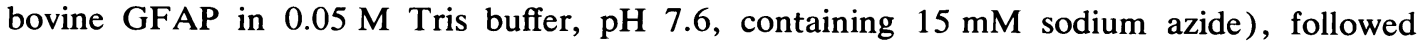
by successive 10-minute incubations with biotinylated link antibody (anti-rabbit and antimouse immunoglobulins in PBS, containing carrier protein and $15 \mathrm{mM}$ sodium azide) and peroxidase-labelled streptavidin. Immunostaining ended after a 10-minute incubation with substrate chromagen solution (3\% 3-amino-9-ethylcarbazole in N,N-dimethylformamide).

For negative control, sections were incubated with nonimmune rabbit serum instead of primary antibody. Moreover, positive controls were provided by sepcimens of normal human brain. 


\section{Results}

From 13 to $22 \mathrm{GW}$, GFAP immunostaining could not be demonstrated anywhere in the retina. At $24 \mathrm{GW}$, the GFAP immunohistochemistry weakly labelled astrocytes and perivascular glia in the nerve fiber and ganglion cell layers near the optic disc (Fig. 1A). From 25 to $28 \mathrm{GW}$, vessels, especially those in the nerve fiber layer, were invested with GFAPpositive perivascular elements (Fig. 1B), and glial foot process also showed immunoreactivity. At locations distant from the optic disc GFAP staining was confined to perivascular elements and immature astrocytes in the nerve fiber layer. Between 30 and $37 \mathrm{GW}$, GFAPpositive perivascular glial cells were strongly immunoreactive (Fig. 1C). At $40 \mathrm{GW}$ the staining pattern was similar to that described at 30 to $37 \mathrm{GW}$, with a slight increase in the concentration of GFAP-positive perivascular glia (Fig. 1D) and vascular end-feet at a greater distance from the optic disc. From 4 months onward (Figs. 1E, F), there was no significant change in the pattern of perivascular GFAP immunostaining. Most of the astrocytic processes terminated upon vessels within the nerve fiber and ganglion cell layers, and occasionally upon the inner portion of the inner plexiform layer. GFAP-positive perivascular elements were visualized at sites distant from the optic disc, but GFAP immunoreactivity of perivascular glia in the vicinity of the optic disc was so intense that the structural details were difficult to observe.

\section{Discussion}

Several glial markers are now available and among them GFAP, vimentin, galactocerebroside, 08 antigen, and S-100 protein are widely used to study the presence and location of neuroglial cells in the retina. S-100 protein labels astrocytes and ganglion cells but not the perivascular glia in the human retina (unpublished data). Findings oft he present study indicate that the use of anti-GFAP antibody as a glial marker is helpful to demonstrate GFAP-positive perivascular glial cells and astrocytic end-feet in both the fetal and adult retina.

Human retinal vessels wer devoid of GFAP-positive cells before $24 \mathrm{GW}$. As the retina developed, GFAP staining increased until full term. In the mature retina, vesels in the nerve fiber and inner portion of the inner plexiform layer were fully coverd with a GFAP-positive sheath. This sheath was thickest around large vessels int he vicinity of the optic disc and became thinner with decreasing caliber of vessels and increasing distance from the optic disc. The GFAP immunoreactivity was found in the end-feet of astrocytes and perivascular glial cels, and their processes.

Müller cell processes are reported to terminate on blood vessels in the nerve fiber and findings are not compatible with these reportsganglion cell layers of the rat retina ${ }^{23,24)}$. Our because in the present study the processes of astrocytes which terminate in sucker-like end-feet upon retinal vessels were labelled by anti-GFAP antibody. It has been suggested that in the monkey and human retina the astrocytic processes that join with the fascicles of the nerve fiber layer may be rich in filaments while the processes of the same cell which join with the vessels may be devoid of filaments ${ }^{24,25)}$. In the retina of the albino rat, it has been shown that the astrocytic end-feet upon retinal capillaries are devoid of filaments ${ }^{26}$. Our findings of GFAP immunoreactivity in the vascular end-feet suggest that in the human retina, this pattern may be rare. The perivascular glial cells described in the human retina 

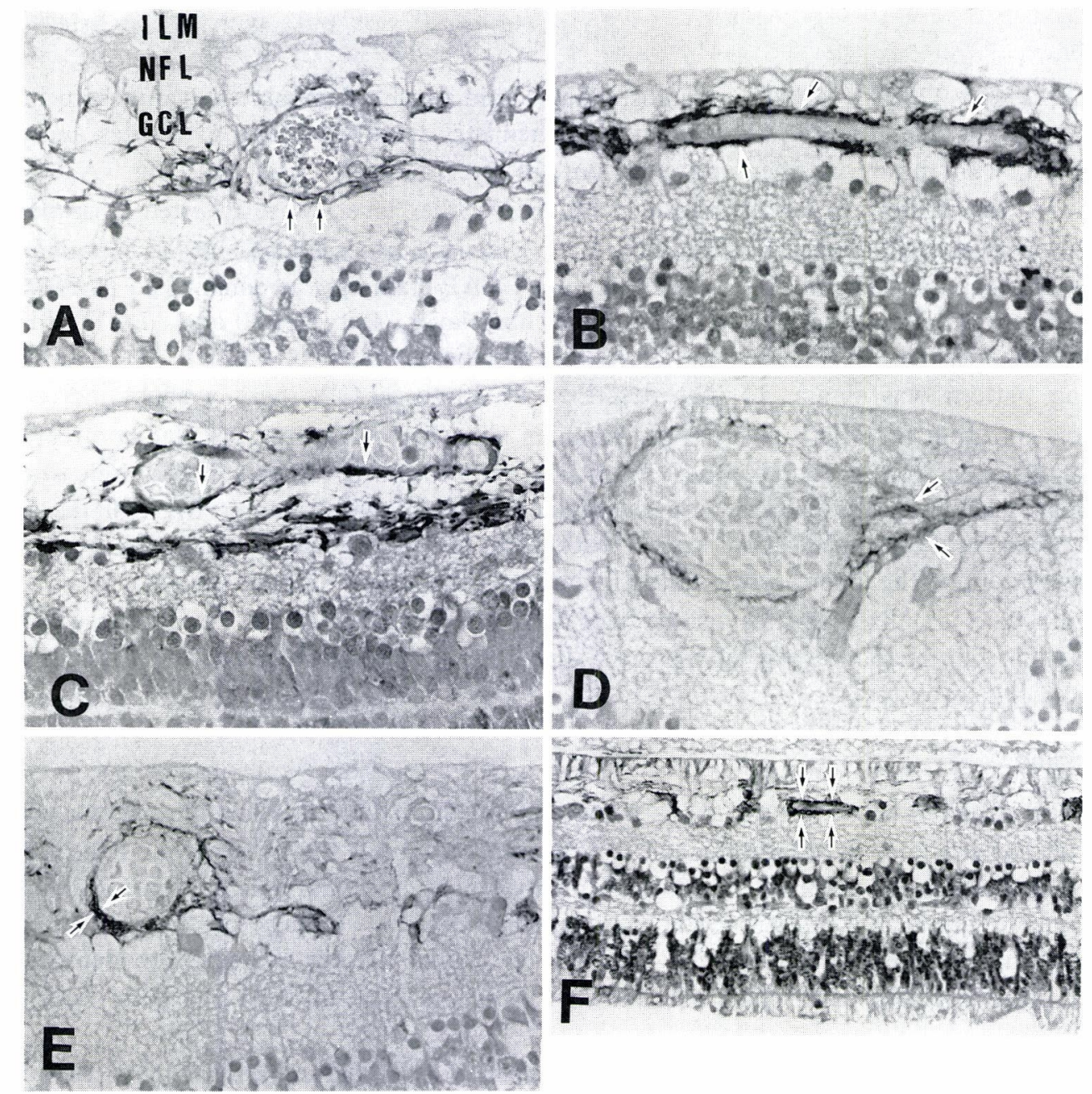

Fig. 1. A. Weakly labelled GFAP-positive perivascular glial cells near the optic disc at $24 \mathrm{GW}$ (arrows). ILM, inner limiting membrane; NFL, nerve fibers layer; GCL, ganglion cell layer. (GFAP, $\times 400)$.

B. GFAP-reactive perivascular glial cells at $28 \mathrm{GW}$ near the optic disc (arrows). (GFAP, $\times 400)$.

C. GFAP-positive perivascular glial cells are seen at $30 \mathrm{GW}$ near the optic disc (arrows). (GFAP, $\times 400)$.

D. At $40 \mathrm{GW}$ the sucker-like end-feet are seen on a retinal vessel in the innermost layers oft he retina approximately midway between the optic disc and he ora serrata (arrows). (GFAP, $\times 400)$.

E. At 4 months GFAP-immunoreactive perivascular glial cells are evident around a vessel midway between the optic disc and the ora serrata (arrows). (GFAP, $\times 400)$.

F. In the adult retina GF AP-immunoreactive perivascular glial cells are evident near the ora serrata (arrows). (GFAP, $\times 200)$. 
in our experiment may be compared with those reported in previous studies ${ }^{2,27)}$.

In summary, we have demonstrated that GFAP-labelled perivascular glial cells are first observed at the posterior pole of the globe and spread anteriorly with increasing age. Further immunohistochemical investigations may contribute to a closer understanding of the location of perivascular glial cells in the human retina.

\section{References}

1) Wolter JR: The astroglia of the human retina and other glial elements of the retina under normal and pathologic conditions. Am J Ophthalmol, 40(II) : 88-99 (1955)

2) Wolter JR: Perivascular glia of the blood vessels of the human retina. Am J Ophthalmol, 44: 766-773 (1957)

3) Bairati A and Bartoli E: Ricerche morfologishe ed istochimiche sulla glia del nevrasse di vertebrati II. Uccelli Zeitsch Zell Bd, 42S: 273-304 (1955)

4) Tao-Cheng JH and Brightman MW: Development of membrane interaction between brain endothelial cells and astrocytes in vitro. Int J Dev Neurosci, 6: 25-87 (1988)

5) Risau W: Differentiation of blood-brain barrier endothelium. News in Physiological Sciences, 4: 151-153 (1989)

6) Bignami A, Eng LF, Dahl D and Uyeda CT: Localization of the glial fibrillary acidic protein in astrocytes by immunofluorescence. Brain Res, 43: 429-435 (1972)

7) Martinez-Ernandez A, Bell KP and Norenberg MD: Glutamine synthetase: glial localization in brain. Science, 195: 1356-1358 (1977)

8) Bignami A, Raju T and Dahl D: Localization of vimentin, the nonspecific intermediate filament protein, in embryonal glia and early dierentiating neurones. Dev Biol, 91 : 286-295 (1982)

9) Ramirez JM, Triviño A, Ramirez AI, Salazen JJ and Garcia-Sanchez J: Immunohistochemical study of human retinal astroglia. Vision Res, 34(15): 1935-1946 (1994)

10) Miller RH and Raff MC: Fibrous and protoplasmic astrocytes are biochemically and developmentally distinct. J Neurosci, 4: 585-592 (1984)

11) Tatsuno I and Arimura A: Pituitary adenylate cyclase-activating polypeptide (PACAP) mobilizes intracellular free calcium in cultured rat type-2, but not in type-1 astrocytes. Brain Res, 662: 1-10 (1994)

12) Eng LF: Glial fibrillary acidic protein (GFAP): The major protein of glial intermediate filaments in differentiated astroctyes. J Neuroimmunol, 8: 203-214 (1985)

13) Seiler MJ and Aramant RB: Photoreceptor and glial markers in human embryonic retina and in human embryonic retinal transplants to rat retina. Dev Brain Res, 80: 81-95 (1994)

14) Linser PJ and Perkins $M$ : Gliogenesis in the embryonic avian optic tectum: neuronal-glial interactions influence astroglial phenotype maturation. Dev Brain Res, 31: 277-290 (1987)

15) Robinson SR and Dreher Z: Evidence for three morphological classes of astroctye in the adult rabbit retina: functional and developmental implications. Neurosci Lett, 106: 261-268 (1989)

16) Cardone B and Roots RI: Comparative immunohistochemical study of glial filament proteins (glial fibrillary acidic protein and vimentin) in goldfish, octopus and snail. Glia, 3: 180-192 (1990)

17) Monzon-Mayor M, Yanes C, Ghandour MS, De Barry J and Gombos G: Glial fibrillary acidic protein and vimentin immunohistochemistry in the developing and adult midbrain of the lizard "Gallotia galloti”. J Com Neurol, 295: 569-579 (1990)

18) Cameron RS and Rakic P: Glial cell lineage in the cerebral cortex. A review and synthesis. Glia, 4: $124-137$ (1991)

19) Eng LF and Bigbee JW: Immunohistochemistry of nervous system specific antigens. In: Advances in Neurochemistry, Vol. 3. Plenum, New York, pp 43-98 (1978)

20) Eng LF: The glial fibrillary acidic (GFA) protein. In: Proteins of the Nervous System, 2nd ed, Raven, New York, pp 85-117 (1980)

21) Dixon RG and Eng LF: Glial fibrillary acidic protein in the retina of the developing albino rat: An immunoperoxidase study of paraffin-embedded tissue. J Comp. Neurol, 195: 305-321 (1981)

22) Vaquera-Orte J, Moral JR and Marion-Giron F: Immunocytochemical demonstration of glial fibrillary acidic protein in the cortical perivascular glial sheat of the cat. Anat $A n z, 163(1)$ : 33-36 
(1987)

23) Hogan MJ and Feeney L: The ultrastructure of the retinal blood vessels (I): The large vessels. J Ultrastruct Res, 9: 10-28 (1963)

24) Hogan MJ and Feeney L: The ultrastructure of the retinal vessels (III): Vascular-glial relationships. J Ultrastruct Res, 9: 47-64 (1963)

25) Hogan MJ and Feeney L: Ultrastructure of Müller cells and perivascular glia. Invest Ophthalmol, 2: 101 (1963)

26) Kissen AT and Bloodworth JMB: Ultrastructure of retinal capillaries of the rat. Exp Eye Res, 1: 1-4 (1961)

27) Liss L: Astroglia and perivascular structures of the human olfactory bulb: A study with silver carbonate. Otol Rhinol Laryngol, 64: 937-944 (1956)

[Received May 28, 1996: Accepted July 23, 1996] 